\title{
Have the Pilot County Hospitals' Service Capability Been Improved Since the Healthcare Reform? An Analysis of 370 Hospitals in China
}

\author{
Yinhong Dong ${ }^{1}$, Xingyi YANG ${ }^{2}$, *Pengqian FANG ${ }^{2}$, Zhengqiong PAN ${ }^{2}$, Zhenni LUO ${ }^{3}$ \\ 1. School of Management, South-Central University for Nationalities, Wuhan, China \\ 2. School of Medicine and Health Management, Tongii Medical College, Huazhong University of Science \& Technology, Wuban, China \\ 3. School of Health Management, Guangzhou Medical University, Guangzhou, China \\ *Corresponding Author: Email: yangxingyi4@163.com
}

(Received 10 Nov 2017; accepted 22 Feb 2018)

\begin{abstract}
Background: County public hospital reform is one of the major tasks proposed in Chinese Healthcare Reform., and the evaluation of hospital reform effectiveness is very important and beneficial since it helps the government to understand the current situation of pilot county public hospitals and smoothly start the reform in all county hospitals.

Methods: This study used hospitals data from 2009 to 2012 to evaluate the effectiveness of county public hospital reform through comprehensive service capability. Descriptive analysis method was used, and factor analysis method was used to extract the main factors associated with service capabilities as well as to calculate a composite score. The t-test of two independent-samples methods was used to comparison analyze.

Results: The differences of common factor scores (hospital scale and service capacity, treatment quality, service quality, and services efficiency) between pilot and non-pilot hospitals were not statistically significant $(P>0.05)$. The service capability score in 2012 was better than that in 2009 either in pilot or non-pilot group $(P<0.05)$. The pilot hospitals' service capability score was better than that in non-pilot groups either in 2010 or $2012(P<0.05)$. However, the differences from 2009 to 2012 of service capability score between pilot and nonpilot hospitals were not statistically significant.

Conclusion: The comprehensive service capability of both pilot and non-pilot group all got improvement. However, county public hospital reform did not significantly play a due good role in improving the service capability in pilot group. The reform was helpful to improve the hospital current situation, but it has not completely achieved policy objectives in the sample hospitals of this study.
\end{abstract}

Keywords: County hospitals; Service capability; Evaluation; Healthcare reform; China

\section{Introduction}

China has the largest population in the world, and over $50 \%$ of its population lives in rural areas. Therefore, the Chinese government must improve the health status of its rural population (1). A three-tiered medical system (county-townvillage) was established in the rural areas of Chi- na. At the village level, which is the bottom of the three-tiered medical system, rural doctors ("barefoot doctors") provide basic curative and preventive medical services for villagers in their own clinics. County hospitals are also responsible for providing training and guidance to township 
health centers, in addition to the supervision of countywide health services (2). In 2012, China had 10940 county hospitals, 37000 township health centers, and 653000 village clinics. Every county has at least one county hospital (3) and almost the entire rural population has access to essential health services at a reasonable cost through the three-tiered medical system.

Before 2009, dwindling financial support from the government caused many rural health facilities to suffer from financial hardships, causing them to spend very little on the purchase of medical equipment and training for health workers (4). Certain market elements forced many of the better-trained health workers to move from rural facilities to urban areas (5), leading to a decline in the service capability of rural health facilities. Increasing distrust and chronic underutilization of rural health clinics occurred. Restricted access to urban medical services further highlighted social issues in the country. Using the occupancy rate as an example, the rate in urban hospitals averaged $85 \%-90 \%$ but averaged less than $50 \%$ in county hospitals $(1,6)$. The differences in resource allocation in rural and urban areas increased. Moreover, while the number of beds and health professionals increased remarkably in urban hospitals, these numbers declined noticeably in rural hospitals over the same period (1). As inequity and the huge health gap became increasingly significant, the Chinese government had to improve its health sector.

In Mar 2009, the Chinese government has launched formally Healthcare Reform with an aim of establishing a more equitable health system (7) and a focus on gradually achieving the goal, for example, everyone has access to basic medical care and health services, as well as improving medical and health services at the grassroots level. County hospitals are essential to the reform as the main provider of healthcare for rural population and as the top tier in the threetiered medical system (8).

Hundreds of county-level public hospitals were selected to participate in a pilot project by Chinese National Ministry of Health and government. Some of the main measures of this pilot project included clearly stated roles and functions of county public hospitals, increased government investment, and the establishment of coordinated, unified and effective administrative mechanisms. Governments at all levels increased health investment of each county to improve infrastructure, health workforce, medical equipment, discipline construction, salary and reward of medical workers, basic pharmaceuticals policy, basic medical insurance and so on.

In China, the effectiveness of the reform evaluated from different perspectives. Some studies evaluated the effectiveness through operational efficiency and productivity of county hospital and showed those have gone down (9-11). The effectiveness through patient's medical expenses and cost of medicine evaluated and those have fallen to some extent as health reform starts $(12,13)$. Some studies evaluated the effectiveness through patients' satisfaction $(14,15)$ and medical staffs' comments about the reform (16,17). Patients' satisfaction was not throughout improved with the implementation of the reform, and some medical staffs hold negative comments about the reform effectiveness in pilot county hospitals. Some studies evaluated the effectiveness through other various variables, such as the number of beds, service infrastructure development, workforce development, medical service quality and efficiency (18-26).

However, there was almost no effectiveness evaluation through comprehensive service capability of public county hospitals before and after the reform. Enhancing the service capability of public county hospitals was one of the key goals pursued by policy makers to ensure that the majority of the rural population would be guaranteed access to basic health services and have common illnesses be treated locally at the rural healthcare level. Therefore, building a comprehensive service capability evaluation index system would assist county hospital reform and development. This study aimed to find the conditions of service capability, evaluate the improvement of service capability of pilot county hospitals after the healthcare reform in China. It also can help the government to understand the current situation 
of pilot county public hospitals and smoothly start the reform in all public hospitals.

\section{Methods}

The study did not involve human participants, specimens or tissue samples, or vertebrate animals, embryos or tissues. The Ministry of Health approved this retrospective study and granted permission to the analysis of data.

\section{Sampling}

By considering both economic and social conditions, 18 representative provinces or cities were chosen across the country for county hospital reform by Chinese Ministry of Health, with 185 pilot county hospitals from 18 provinces or cities selected as an experimental group by county hospital reform policy and 185 non-pilot hospitals selected as a control group by research group. Selection criteria for the control group are as follows: we chose one pilot hospital as a reference and also considered the geographic proximity, similar size, and per capita GDP closest to the specific pilot hospital in 2009. This study has a total sampling of 370 county-level hospitals.

We collected data from the Chinese Ministry of Health for the 370 county hospitals from 2009 to 2012 and included indicators, such as hospital bed size, number of medical staff, workload of hospital departments, hospital efficiency, and quality of medical care.

\section{Methods of data analysis}

Through literature analysis (18-26) and expert consultation, and after considering the availability of data, we screened the related indicators with county hospital service capabilities. The descriptive analysis method was used; factor analysis method was used to extract the main factors associated with service capabilities of county hospitals and to calculate a composite score of the serviceability of county hospitals. The $t$ test of two independent-samples methods was used to comparison analyze.

\section{Service capability indicators of county hospitals}

Eleven indicators related to the service capabilities of county hospitals were selected through literature analysis and expert consultation. These indicators include the actual number of open beds (X1), number of medical staff (X2), number of outpatient and emergency patients per year (X3), number of operations per year (X4), number of discharged patients per year (X5), cure rate (X6), improvement rate (X7), clinical and pathological diagnostic accuracy rate (X8), outpatient and hospital admission diagnostic accuracy rate (X9), bed turnover rate (X10), and average length of stay (X11). All statistical indicators are explained below:

1) Actual number of open beds (X1): the total number of sickbeds opened in a hospital.

2) Number of medical staff (X2): the total number of medical staff in a hospital

3) Number of outpatient and emergency patients (X3): the number of non-hospitalized patients is a summation of the number of outpatient and emergency patients.

4) Number of operations per year (X4): the total number of operations performed in a hospital in one year.

5) Number of discharged patients (X5): all patients discharged from the hospital, including all people listed in the "cured," "improvement," and "not cured" groups after treatment.

6) Cure rate: the frequency of patients who become healed after treatment (X6). Cure rate $=$ (number of cured cases/number of discharged patients) $\times 100 \%$.

7) Improvement rate $(\mathrm{X} 7)=$ (number of improved cases/number of discharged patients) $\times$ $100 \%$.

8) Clinical and pathological diagnostic accuracy rate(X8): (number of clinical and pathological diagnosis accuracy/cases of pathological diagnosis) $\times 100 \%$.

9) Outpatient and hospital admission diagnostic accuracy rate $(X 9)$ : (number of outpatient and inpatient diagnosis/number of inpatients) $\times 100 \%$.

10) Bed turnover rate $(\mathrm{X} 10)$ : the number of discharged patients for each bed in a period. According to the National Health Statistical Investigation System (2007), the formula for bed turnover rate is the number of discharged patients / average number of open beds. 
11) Average Length of Stay (ALOS) (X11): the average length of hospital stay for each discharged patient is a comprehensive indicator used in the evaluation of benefit and efficiency, as well as healthcare quality and technological level. "Average length of stay=Total bed days occupied by discharged patients/number of discharged patients."

\section{Process of factor analysis}

We included indices X1 to X11 in 2009 and 2012 of the 370 county hospitals into the factor analysis.

\section{KMO and Bartlett's Test}

The KMO test coefficient $=0.801>0.5$. The significant probability $P$-value of chi-square statistic of Bartlett test is $<0.001$, the data are highly suitable for factor analysis (Table 1).

\section{Extracting characteristic roots}

With characteristic roots $>1$ as a standard, four characteristic roots were extracted, the cumulative contribution rate of the former four main factors was $73.442 \%$, indicating that the amount of original data has been extracted by $73.442 \%$. Moreover, the original data can be reflected more objectively by the four main factors (Table 2).

\section{Extracting the common factor}

The typical representative variables of the factor loading matrix were not very prominent and thus were difficult to explain well. A rotated factor model can be built after maximum variance orthogonal rotation. This model shows that the first main factor F1 had higher loads on variables X1 (actual number of open beds), X2 (number of medical staff), X3 (number of outpatient and emergency patients per year), X4 (the number of operations per year), and $\mathrm{X} 5$ (number of discharged patients), which were $0.887,0.883,0.842,0.811$, and 0.744 , respectively. The results showed that $\mathrm{F} 1$ reflected the situations of $\mathrm{X} 1, \mathrm{X} 2, \mathrm{X} 3, \mathrm{X} 4$, and X5; F1 was labeled the hospital scale and service quantity factor. The second main factor F2 (treatment quality factor) had higher loads on variables X6 (cure rate) and X7 (improvement rate); the former was -0.957 and the latter was 0.953 . $\mathrm{F} 2$ reflected the situations of $\mathrm{X} 6$ and $\mathrm{X} 7$. The third main factor, F3, had higher loads on variables X8 (clinical and pathological diagnostic accuracy rate) and X9 (outpatient and hospital admission diagnostic accuracy rate), which were 0.826 and 0.824 , respectively.

Table 1: KMO and Bartlett Test

\begin{tabular}{lcc}
\hline Kaiser-Meyer-Olk in Measure of Sampling Adequacy & & $\boldsymbol{0 . 8 0 1}$ \\
\hline Bartlett's Test of Sphericity & Approx. Chi-Square & 15044.876 \\
& Df & 55 \\
& Sig. & 0.000 \\
\hline
\end{tabular}

Table 2: Total Variance Explained

\begin{tabular}{|c|c|c|c|c|c|c|c|c|c|}
\hline \multirow{2}{*}{ 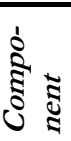 } & \multicolumn{3}{|c|}{ Initial eigenvalues } & \multicolumn{3}{|c|}{ Extracted square and loaded } & \multicolumn{3}{|c|}{ Rotating square and loaded } \\
\hline & Total & $\begin{array}{c}\% \text { of } \\
\text { Variance }\end{array}$ & $\begin{array}{c}\text { Accumulative } \\
\%\end{array}$ & Total & $\begin{array}{c}\% \text { of } \\
\text { Variance }\end{array}$ & $\begin{array}{c}\text { Accumu- } \\
\text { lative } \%\end{array}$ & Total & $\%$ of Variance & $\begin{array}{c}\text { Accumula- } \\
\text { tive } \%\end{array}$ \\
\hline 1 & 4.221 & 38.369 & 38.369 & 4.221 & 38.369 & 38.369 & 3.647 & 33.152 & 33.152 \\
\hline 2 & 1.575 & 14.320 & 52.689 & 1.575 & 14.320 & 52.689 & 1.955 & 17.770 & 50.922 \\
\hline 3 & 1.257 & 11.429 & 64.118 & 1.257 & 11.429 & 64.118 & 1.404 & 12.761 & 63.684 \\
\hline 4 & 1.026 & 9.324 & 73.442 & 1.026 & 9.324 & 73.442 & 1.073 & 9.758 & 73.442 \\
\hline 5 & .918 & 8.349 & 81.791 & & & & & & \\
\hline 6 & .623 & 5.664 & 87.455 & & & & & & \\
\hline 7 & .559 & 5.081 & 92.536 & & & & & & \\
\hline 8 & .327 & 2.972 & 95.508 & & & & & & \\
\hline 9 & .242 & 2.200 & 97.708 & & & & & & \\
\hline 10 & .175 & 1.590 & 99.297 & & & & & & \\
\hline 11 & .077 & .703 & 100.000 & & & & & & \\
\hline
\end{tabular}

Extraction Method: Principal Component Analysis 
F3 was called the diagnostic quality of hospital service quality factor. F3 reflected the situations of X8 and X9. The fourth main factor, F4, had higher loads on variables X10 (bed turnover times) and X11 (average length of stay) at 0.894 and -0.449 , respectively. F4 reflected the situations of X10 and $\mathrm{X} 11$; F4 was called hospital services efficiency factor (Table 3).

\section{Build factor score function, and to calculate factor scores}

The coefficient matrix of factor score is shown in

Table 4. The common factor is expressed as a linear combination of the original variables. A linear regression method was used to obtain the least squares coefficient estimates, namely the factor score coefficients. We calculate factor scores with factor score coefficients by using the following formula (Table 4).

We establish the factor score function as follows: $\mathrm{F} 1=0.256 \mathrm{X} 1+0.250 \mathrm{X} 2+0.231 \mathrm{X} 3+0.242 \mathrm{X} 4+0.24$ $5 \times 5+0.085 \times 6-0.082 \times 7-0.059 \times 8-$

$0.019 \times 9+0.089 \times 10+0.027 \times 11$ $\mathrm{F} 2=-0.045 \times 1-0.025 \times 2-0.022 \times 3-0.083 \times 4-$ $0.072 \times 5-0.539 \times 6+0.535 \times 7-0.013 \times 8-$ $0.080 \times 9+0.042 \times 10+0.010 \times 11$

Table 3: rotated component matrix

\begin{tabular}{lllll}
\hline Variable & \multicolumn{3}{l}{ Component } & \\
\hline & 1 & 2 & 3 & 4 \\
X1 number of open beds & .887 & .153 & .057 & -.106 \\
X2 number of medical staff & .883 & .185 & .062 & -.099 \\
X3 number of outpatient and emergency patients per year & .842 & .187 & .085 & -.142 \\
X4 number of operations per year & .811 & .073 & .071 & -.097 \\
X5 number of discharged patients & .744 & .053 & .015 & .133 \\
X6 cure rate & -.187 & -.957 & -.076 & .046 \\
X7 improvement rate & .193 & .953 & .086 & -.035 \\
X8 clinical and pathological diagnostic accuracy rate & .025 & .111 & .826 & -.016 \\
X9 outpatient and hospital admission diagnostic accuracy rate & .110 & .021 & .824 & -.024 \\
X10 bed turnover rate & .078 & .043 & .046 & .894 \\
X11 average length of stay & .263 & .121 & .092 & -.449 \\
Extraction method: principal component analysis. & & & & \\
Rotation method: varimax with Kaiser normalization. & & & & \\
A. Rotation to converge in four iterations. & & & & \\
\hline
\end{tabular}

Table 4: Coefficient matrix of component scores

\begin{tabular}{|c|c|c|c|c|}
\hline \multirow[t]{2}{*}{ Variable } & \multicolumn{4}{|c|}{ Components } \\
\hline & 1 & 2 & 3 & 4 \\
\hline X1 Number of open beds & .256 & -.045 & -.026 & -.024 \\
\hline X2 Number of medical staff & .250 & -.025 & -.025 & -.016 \\
\hline X3 Number of outpatient and emergency patients per year & .231 & -.022 & -.006 & -.061 \\
\hline X4 Number of operations per year & .242 & -.083 & -.003 & -.024 \\
\hline X5 Number of discharged patients & .245 & -.072 & -.036 & .190 \\
\hline X6 Cure rate & .085 & -.539 & .043 & -.005 \\
\hline X7 Improvement rate & -.082 & .535 & -.035 & .017 \\
\hline X8 Clinical and pathological diagnostic accuracy rate & -.059 & -.013 & 609 & .003 \\
\hline X9 Outpatient and hospital admission diagnostic accuracy rate & -.019 & -.080 & .610 & -.001 \\
\hline X10 Bed turnover times & .089 & .042 & .040 & .870 \\
\hline X11Average length of stay & .027 & .010 & .035 & -.406 \\
\hline \multicolumn{5}{|l|}{ Extraction method: principal component analysis. } \\
\hline Rotation method: varimax with Kaiser normalization. & & & & \\
\hline
\end{tabular}


$\mathrm{F} 3=-0.026 \mathrm{X} 1-0.025 \mathrm{X} 2-0.006 \mathrm{X} 3-0.003 \mathrm{X} 4-$

$0.036 \times 5+0.043 \times 6-$

$0.035 \times 7+0.609 \times 8+0.610 \times 9+0.040 \times 10+0.035 \times 11$

$\mathrm{F} 4=-0.024 \mathrm{X} 1-0.016 \mathrm{X} 2-0.061 \mathrm{X} 3-0.024 \mathrm{X} 4+0.190 \mathrm{X} 5-$

$0.005 \times 6+0.017 \times 7+0.003 \times 8-0.001 \times 9+0.870 \times 10-$

$0.406 \mathrm{X} 11$

The original data of X1 to X11 were standardized values using SPSS (Chicago, IL, USA).

\section{Calculated score of comprehensive service capabilities}

In Table 2 "Total Variance Explained," the four common factor scores were weighted sums and the weight referred to each factor's variance contribution rate. The variance contribution rates of the four common factors (F1, F2, F3, and F4) were $33.152 \%, 17.770 \%, 12.761 \%$, and $9.758 \%$, respectively. The total variance contribution rate of the four common factors was $73.442 \%$. The comprehensive score function for services capability is as follows:

$\mathrm{F}=(33.152 \% \mathrm{~F} 1+17.770 \% \quad \mathrm{~F} 2+12.761 \% \quad \mathrm{~F} 3$ $+9.758 \% \mathrm{~F} 4) / 73.442 \%$.

\section{Results}

\section{Common factor scores in pilot and non-pilot hospitals}

The common factor scores are shown in Table 5. F1 represents the hospital scale and service quantity factor. F2 refers to the treatment quality factor. F3 represents the diagnostic quality factor. F4 was called the hospital services efficiency factor. " 0 " represents the average level of the study samples, a score larger than 0 indicates above average level, and a negative value represents below average level. Because of the characteristics of the factor analysis method, data have been standardized before calculating the factor scores (Table 5).
The comparing results on common factor scores between pilot and non-pilot hospitals are shown in Table 6. For F1, the (mean $\pm \mathrm{SD}$ ) of the different values of factor score between 2009 and 2012 were $0.4142 \pm 0.3520$ (pilot group) and $0.3468 \pm 0.4624$ (non-pilot group). The difference between 2009 and 2012 was normally distributed within each group and the two population variances were equal at the significant level 0.05 $(\mathrm{F}=0.002, P=0.964)$. The t-test of two independent-samples resulted in $\mathrm{t}=1.579, P=0.115$.

For F2, the (mean $\pm \mathrm{SD}$ ) s of the different values of factor score between 2009 and 2012 were $0.0704 \pm 0.5945$ (pilot group) and $0.0739 \pm 0.6602$ (non-pilot group). The difference between 2009 and 2012 was normally distributed within each group and the two population variances were equal at the significant level $0.05 \quad(\mathrm{~F}=0.187$, $P=0.666$ ). The t-test of two independent-samples resulted in $\mathrm{t}=-0.053, P=0.958$.

For F3, the (mean $\pm \mathrm{SD}$ ) s of the different values of factor score between 2009 and 2012 were $0.0126 \pm 0.4905$ (pilot group) and $0.0472 \pm 0.4523$ (non-pilot group). The difference between 2009 and 2012 was normally distributed within each group and the two population variances were equal at the significant level 0.05 ( $\mathrm{F}=0.007$, $P=0.935)$. The t-test of two independent-samples resulted in $\mathrm{t}=-1.219, P=0.224$.

For F4, the (mean $\pm \mathrm{SD}$ ) s of the different values of factor score between 2009 and 2012 were $0.1027 \pm 0.3043$ (pilot group) and $0.0800 \pm 0.4080$ (non-pilot group). The difference between 2009 and 2012 was normally distributed within each group and the two population variances were equal at the significant level $0.05 \quad(\mathrm{~F}=0.372$, $P=0.542)$. The t-test of two independent-samples resulted in $\mathrm{t}=0.606, P=0.545$.

Table 5: Descriptive statistics for common factors (F1, F2, F3, F4) scores between pilot and non-pilot hospitals $(\bar{x} \pm$ SD)

\begin{tabular}{lcccc}
\hline $\begin{array}{l}\text { Common } \\
\text { factors }\end{array}$ & Polit & & Non-pilot & \\
\hline & & 2012 & 2009 & 2012 \\
F1 & 2009 & $0.5300 \pm 1.2446$ & $-0.1262 \pm 0.9542$ & $0.1136 \pm 0.6179$ \\
F2 & $0.1158 \pm 0.9671$ & $0.1367 \pm 1.0007$ & $-0.0530 \pm 1.0373$ & $0.0210 \pm 1.0807$ \\
F3 & $0.0663 \pm 0.9846$ & $0.0344 \pm 0.7468$ & $-0.0432 \pm 0.9116$ & $0.0040 \pm 0.9579$ \\
F4 & $0.0470 \pm 0.6440$ & $0.0337 \pm 0.3981$ & $-0.0180 \pm 0.5594$ & $0.0620 \pm 0.5738$ \\
\hline
\end{tabular}


Table 6: The comparing result on common factor scores between pilot and non-pilot hospitals

\begin{tabular}{lcccc}
\hline Common factors & Group & $\bar{d}_{2012-2009} \pm S_{d}$ & $\boldsymbol{t}$ & $\boldsymbol{P}$ \\
\hline F1 & Polit & $0.4142 \pm 0.3520$ & 1.579 & 0.115 \\
& Non-pilot & $0.3468 \pm 0.4624$ & 0.958 \\
F2 & Polit & $0.0704 \pm 0.5945$ & -0.053 & 0.224 \\
F3 & Non-pilot & $0.0739 \pm 0.6602$ & -1.219 & 0.545 \\
& Polit & $-0.0126 \pm 0.4905$ & & 0.606 \\
F4 & Non-pilot & $0.0472 \pm 0.4523$ & & \\
& Polit & $0.1027 \pm 0.3043$ & & \\
\hline
\end{tabular}

Comprehensive service capabilities scores in pilot and non-pilot hospitals

The calculation results of the comprehensive factor scores (F) of the service capabilities are shown in Table 7. "O" represents the average level of the study samples, whereas a negative value represents below average level. In pilot and nonpilot hospital, In pilot hospitals, the F scores were normally distributed both in 2009 and in 2012. The two population variances were not equal at the significant level 0.05 ( $\mathrm{F}=6.217, P=0.013)$. The correction t-test of two independent-samples resulted in $\mathrm{t}=-3.662, P=0.000$.

In non-pilot hospitals, the F scores were normally distributed both in 2009 and in 2012. The two population variances were equal at the significant level $0.05(\mathrm{~F}=3.423, P=0.065)$. The t-test of two independent-samples resulted in $\mathrm{t}=-3.270$, $P=0.001$.

In 2009, the F scores were normally distributed both in pilot and non-pilot hospitals. The two population variances were equal at the significant level 0.05 ( $F=1.071, P=0.301)$. The t-test of two independent-samples resulted in $\mathrm{t}=0.367$, $P=0.006$.

In 2012, the F scores were normally distributed both in pilot and non-pilot hospitals. The two population variances were equal at the significant level $0.05(\mathrm{~F}=1.350, P=0.246)$. The t-test of two independent-samples resulted in $\mathrm{t}=2.639$, $P=0.009$.

The comparing results on comprehensive service capabilities scores (F) between pilot and nonpilot hospitals are shown in Table 8. For F, the (mean $\pm \mathrm{SD}$ ) $\mathrm{s}$ of the different values of factor score between 2009 and 2012 were $0.2151 \pm 0.1770$ (pilot group) and $0.1930 \pm 0.2523$ (non-pilot group). The difference between 2009 and 2012 was normally distributed within each group and the two population variances were equal at the significant level $0.05 \quad(\mathrm{~F}=1.255$, $P=0.263)$. The t-test of two independent-samples resulted in $\mathrm{t}=0.976, P=0.330$.

Table 7: Comprehensive service capabilities scores (F) from 2006 to 2012

\begin{tabular}{llll}
\hline Group & $\overline{\boldsymbol{x}} \pm \boldsymbol{S D}$ & $\boldsymbol{t}$ & $\boldsymbol{P}$ value \\
\hline Pilot group & $0.0673 \pm 0.5111$ & -3.662 & 0.000 \\
2009 & $0.2828 \pm 0.6158$ & & \\
2012 & $-0.0800 \pm 0.5144$ & -3.27 & 0.001 \\
Non-pilot group & $0.1136 \pm 0.6179$ & & \\
2009 & & & \\
2012 & $0.0673 \pm 0.5111$ & 2.76 & 0.006 \\
2009 & $-0.0800 \pm 0.5144$ & & \\
Pilot group & $0.2828 \pm 0.6158$ & 2.639 & 0.009 \\
Non-pilot group & $0.1136 \pm 0.6179$ & & \\
2012 & & & \\
Pilot group & Non-pilot group & & \\
\hline
\end{tabular}


Dong et al.: Have the Pilot County Hospitals' Service Capability Been ...

Table 8: The comparing result on comprehensive service capabilities scores $(\mathrm{F})$ between pilot and non-pilot hospitals

\begin{tabular}{lllll}
\hline & Group & $\bar{d}_{2012-2009} \pm S_{d}$ & $\boldsymbol{t}$ & P value \\
\hline F & Polit & $0.2151 \pm 0.1770$ & 0.976 & 0.330 \\
& Non-pilot & $0.1930 \pm 0.2523$ & & \\
\hline
\end{tabular}

\section{Discussion}

The differences from 2009 to 2012 of common factor scores (F1, F2, F3, F4) between pilot and non-pilot hospitals were not statistically significant $(\mathrm{P}>0.05)$. There was a difference on the improvement from 2009 to 2012 of F1 (hospital scale and service capacity), F2 (treatment quality), F3 (service quality) and F4 (services efficiency) of sample hospitals between pilot and non-pilot groups. The reform could not have improved fully county public hospitals' scale and service quantity, treatment quality, service quality and services efficiency.

The comprehensive service capabilities score (F) of hospitals in 2012 was better than that in 2009 either in pilot or non-pilot group $(P<0.05)$. Meanwhile, the pilot hospitals' comprehensive service capabilities score $(\mathrm{F})$ was better than that in non-pilot groups either in 2010 or 2012 $(P<0.05)$. The comprehensive service capabilities score $(\mathrm{F})$ of both pilot and non-pilot group all got improvement with the development and progress of socio-economic level, improvement of medical technology and serviceability, and the implementation of the new healthcare reform.

The government increased financial input in the construction of county hospitals, especially the pilot ones. After implementing the new healthcare reform, the coverage of the New Rural Cooperative Medical Scheme, medical insurance for urban workers, and medical insurance for urban residents improved. The medical service demand from the rural population increased and also contributed to the scale expansion of county hospitals. From 2009 to 2012, in both pilot and non-pilot county hospitals, the scale (including number of beds and medical staffs) increased rapidly. The service quantity (including number of outpatient and emergency patients, operations and discharged patients) and the treatment quality (including cure rate and improvement rate) increased. The diagnostic quality (including clinical and pathological diagnostic, outpatient and hospital admission diagnostic) in pilot hospitals decreased, while that in non-pilot hospitals increased. The services efficiency (including bed turnover times, average length of stay) also increased. Judging from the overall trend, the comprehensive service capabilities score $(\mathrm{F})$ of both pilot and non-pilot group all got improvement.

However, the differences from 2009 to 2012 of comprehensive service capabilities score (F) between pilot and non-pilot hospitals were not statistically significant $(P>0.05)$. The county public hospital reform did not significantly play a due good role in improving the service capability in pilot group which is one of the reform's goals. Since China has implemented health care reform in 2009, many studies and papers relating to the reform has published both in Chinese journals and international journals. Most of these papers showed the positive results of the reform. However, this study indicated that the reform has shortcomings. This study evaluated the public hospital reform from a more comprehensive, objective and unique perspective, getting a different point of view.

One of the important reasons was China health reform policy was paying more attention to inputs rather than outputs for pilot hospital and lack of evaluation and supervision on hospital running or operation. Besides, the decisionmaking department of public health made the decision of how much should be in each hospital by experience management without attaching importance to equity and efficiency, as well as quality (27). Governments should ensure that resources are allocated according to need (4). 
Studies in other countries also showed the similar problems. A study analyzing Mexico's health system (28) concluded that the measures implemented to reform the Mexican health system have failed to achieve the intended results; on the contrary, they have led to a reduction in interventions, rising costs, and a decrease in the installed capacity and professional personnel for the system's operation. Health systems face new challenges, inevitably requiring that the analyses be situated in a broader framework. In Sweden (29) in terms of structure, process and outcomes mentioned that resource allocation to $\mathrm{PHC}$ has become more dependent on provider location, $\mathrm{pa}$ tient choice and demand, and less in need of care. This situation needs to be carefully monitored and countered where necessary. In Ghana, Central government regulations for resource allocation and use should be more flexible, to make services better respond to local needs (30).

These experiences and lessons in this study are meaningful and noteworthy for other counties, especially developing countries. The new suggestions for health care system of other countries are as follows: 1) paying more attention to outputs rather than inputs. 2) Fully evaluation and supervision on health system running, and the equity, efficiency, quality of health system. 3) The decision-making department of public health should make the decision of input according to equity and efficiency, as well as quality, rather than by experience management.

\section{Conclusion}

The comprehensive service capability of both pilot and non-pilot group all got improvement. However, county public hospital reform did not significantly play a due good role in improving the service capability in pilot group. The reform was helpful to improve the hospital current situation, but it has not completely achieved policy objectives in the sample hospitals of this study.

\section{Ethical considerations}

Ethical issues (Including plagiarism, informed consent, misconduct, data fabrication and/or falsification, double publication and/or submission, redundancy, etc.) have been completely observed by the authors.

\section{Acknowledgments}

This study was part of the project "Study on the construction and institutional arrangement of national universal health insurance system based on balance of interests and the integration of system", which was funded by the National Natural Science Funds of China (Grant no. 71333005). Sincere thanks to all directors who participated in this study. We are thankful to our colleagues Lu Zhao and Qiuxia Han who provided expertise that greatly assisted the research, although they may not agree with all of the interpretations provided in this paper. We are also grateful to Xiaoyan Zhang for assistance with data analysis, and Rui Min who moderated this paper and in that line improved the manuscript significantly. We have to express our appreciation to the Xue Bai for sharing pearls of wisdom with us during the course of this research.

\section{Conflict of interest}

The authors declare that there is no conflict of interests.

\section{References}

1. Shi L (1993). Health care in China: a rural-urban comparison after the socioeconomic reforms. Bull World Health Organ, 71(0): 723-736.

2. Gai R Y, Zhou C C, Xu L Z, et al (2010). Health resource allocation and productive efficiency of Chinese county hospitals: data from 1993 to 2005. Biosci Trends, 4: 218-224.

3. Developed Statistical Bulletin of National Health and Family Planning in China (2012). National Health and Family Planning Commission of the People's Republic of China.

4. Bloom G, Tang S, Gu X (1995). Financing rural health services in China in the context of economic reform. J Int Dev, 7:423-441. 
5. Bloom $G$, Xingyuan $G$ (1997). Health sector reform: lessons from China. Soc Sci Med, 45:351-360.

6. Cai K (1990). The future directions of the county and township health institutions. Chinese Rural Health Services Management, 10-13.

7. State Council (2009). Guidelines for deepening health system reform.

8. Shen X. Chuanjun Du (2008). China to pilot medical reform plan this year. Chinese Medical Journal, 121:199.

9. Ying L (2015). The operational technical efficiency evaluation of Ningxia county and township hospitals before and after the new medical reform. Shandong University, Jinan.

10. Jiang S, Wu W, Huo H (2015). Study on the efficiency of 41 county-level public hospitals in Guangxi based on DEA model. Chinese Hospital Management, 35:13-15.

11. Shuai J, Weimin W, Li F, et al (2014). Surveying the patients' satisfaction on pilot county hospitals after the new medical reform. Chinese Health Service Management, 12:905-907.

12. Huimei H (2014). Preliminary assessment of comprehensive reform of county public hospital in Zhejiang Province. Zhejiang University, Hangzhou.

13. Zhenfang H (2014). Study and evaluation about the economic operation of the county hospitals in Anhui Province before after the comprehensive reform. Anhui Medical University, Hefei.

14. Suyi L, Chaokun X, Meng B, et al (2013). Healthcare satisfaction among residents in Suqian after healthcare system reform and the influencing factors. Chinese General Practice, 16:84-86.

15. Weiyun L, Yulan J (2014). Survey on the grassroots medical and health service satisfaction of residents in Chengde city after the medical reform. Hebei Medicine, 20:864-867.

16. Fang P, Luo Z, Fang Z (2015). What is the job satisfaction and active participation of medical staff in public hospital reform: a study in Hubei province of China. Hum Resour Health, 13:34.

17. Dongmei $H$, Wenqiang $Y$, Qianqian $Y$ et al (2015). Comparison of work well-being among physicians in public hospitals before and after the new health reform. Chinese Journal of Hospital Administration, 31:217-220.

18. Gross R (2004). A Consumer Based Tool for Evaluating the quality of Health Services in the Israeli
Health Care System Following Reform. Health Policy, 68:143-158.

19. Von demKnesebeck, Joksimovic L, Badura B, Siegri J (2002). Evaluation of a community-level health policy. Health Policy, 61:111-122.

20. Liberati A, Apolone G, Lang T, Lorenzo S (1995). A European project assessing the appropriateness of hospital: background, objectives and preliminary results. Int J Qual Health Cane, 7:187-199.

21. WHO (1996). Integration of Health Care Delivery, Report of WHO Study Group. World Health Organ Tech Rep Ser, 861:1-68.

22. Pai YP, Chary ST (2013). Dimensions of hospital service quality: a critical review: Perspective of patients from global studies. Int $J$ Health Care Qual Assur, 26:308-40.

23. Camilleri D, O'Callaghan M (1998). Comparing public and private hospital care service quality. Int J Health Care Qual Assur Inc Leadersh Health Serv, 11(4-5):127-33.

24. Choi KS, Lee H, Kim C, Lee S (2005). The service quality dimensions and patient satisfaction relationships in South Korea: comparisons across gender, age and types of service. Journal of Senvices Marketing, 19:140-149.

25. Dabholkar PA, Shepherd CD, Thorpe DI (2000). A comprehensive framework for service quality: an investigation of critical conceptual and measurement issues through a longitudinal study. $J$ Retailing, 76:139-73.

26. Dagger T, Sweeney JC (2007). Service quality attribute weights: how do novice and longer-term customers construct service quality perceptions? Journal of Service Research, 10:22-42.

27. Jiang S, Wu W, Fang P (2016). Evaluating the effectiveness of public hospital reform from the perspective of efficiency and quality in Guangxi, China. SpringerPlus, 5:1922.

28. López-Arellano O, Jarillo-Soto EC (2017). A health system's neoliberal reform: evidence from the Mexican case. Cad Saúde Público, 33:e00087416.

29. Bo Burström, Kristina Burström, Gunnar Nilsson, et al (2017). Equity aspects of the Primary Health Care Choice Reform in Sweden - a scoping review. Int J Equity Health, 16:29.

30. Agyepong IA (1999). Reforming health service delivery at district level in Ghana: the perspective of a district medical officer. Health Policy Plan, 14:59-69. 